\title{
Global Nash convergence of Foster and Young's regret testing
}

\author{
Fabrizio Germano \\ Gábor Lugosi \\ fabrizio.germano@upf .edu \\ lugosi@upf .es \\ Departament d'Economia i Empresa \\ Universitat Pompeu Fabra \\ Ramon Trias Fargas 25-27 \\ 08005 Barcelona, Spain
}

October 2004

\begin{abstract}
We construct an uncoupled randomized strategy of repeated play such that, if every player follows such a strategy, then the joint mixed strategy profiles converge, almost surely, to a Nash equilibrium of the one-shot game. The procedure requires very little in terms of players' information about the game. In fact, players' actions are based only on their own past payoffs and, in a variant of the strategy, players need not even know that their payoffs are determined through other players' actions. The procedure works for general finite games and is based on appropriate modifications of a simple stochastic learning rule introduced by Foster and Young [10].

Keywords Regret testing; Regret based learning; Random search; Stochastic dynamics; Uncoupled dynamics; Global convergence to Nash equilibria. JEL Classification C72, C73, D81, D83.
\end{abstract}




\section{Introduction}

We study a simple stochastic learning procedure such that if all players play according to it, then their joint mixed strategy profile will converge, almost surely, to a Nash equilibrium of the game. An important feature of the procedure is that it requires very little in terms of what players need to know about the underlying game. In fact, for a variant of the basic version, we show that players' mixed strategy profiles converge to a Nash equilibrium of the stage game even if players do not know that they are playing a game with other players. What they do need to know are their own payoffs which they need to observe over sufficiently long periods of time.

The procedure is a variant of the "regret testing" learning rule introduced by Foster and Young [10]. The main idea behind this procedure is that time is divided into sufficiently long periods such that at the beginning of each period each player chooses a mixed strategy at random and plays according to the corresponding distribution for the duration of the period. If the player could not have performed much better by playing some other fixed strategy throughout the (just elapsed) period, then he repeats the same mixed strategy for the next period; otherwise the player randomly selects a new mixed strategy and plays it again during the next period. The procedure thus implements a kind of exhaustive search. The basic variation we study adds "experimentation" to Foster and Young's procedure in the sense that, with small probability, players sample a new mixed strategy even if they could not have done much better with any fixed strategy over the elapsed period. Among other things this guarantees that the process of the mixed strategy profiles at the beginning of the given periods is an irreducible Markov chain.

More specifically, the setup is the following. We consider repeated play of a finite $N$-player normal form game. At each time instant $t=1,2, \ldots$, player $i \in N$ chooses a mixed strategy $\sigma_{t}^{i} \in \Sigma_{i}$ depending on the history and selects an action $s_{t}^{i}$ randomly according to the distribution of $\sigma_{t}^{i}, i \in N$. In the basic setup, we assume that after taking an action at time $t$, player $i$ 
observes the actions $s_{t}^{-i}$ played by the rest of the players. (This "standard monitoring" assumption is significantly weakened in Section 7.) However, we focus our attention on uncoupled procedures in the sense that each player $i$ knows his own payoff function $\gamma^{i}$ but ignores the payoff functions of the rest of the players, see Hart and Mas-Colell [21, 22]. We also allow randomized procedures, in the sense that at each time instant $t$ player $i$ has access to a random variable $\chi_{i, t}$ whose value he can use in determining $\sigma_{t}^{i}$ where the $\chi_{i, t}$ are independent and (say) uniformly distributed over the interval $[0,1]$.

Our main objective here is to see whether uncoupled randomized procedures can lead to Nash equilibrium. More precisely, does there exist a randomized uncoupled strategy ${ }^{1}$ such that, regardless of what the underlying game is, if all players follow such a strategy, the joint mixed strategy profiles $\sigma_{t}=\left(\sigma_{t}^{1}, \ldots, \sigma_{t}^{N}\right)$ converge, almost surely, to a Nash equilibrium of the one-shot game. Several examples of uncoupled procedures that lead to equilibrium have been constructed in the literature, though weaker notions of equilibrium and weaker types of convergence have been considered.

Perhaps the first such universal convergence result was shown by Foster and Vohra [7], who proved the existence of adaptive procedures such that the joint empirical frequencies of play

$$
\widehat{P}_{t}(s)=\frac{1}{t} \sum_{\tau=1}^{t} I_{s_{\tau}=s}, \quad s \in S,
$$

converge to the set of correlated equilibria of the game, see also Foster and Vohra [8], Fudenberg and Levine [12, 14], Hart and Mas-Colell [17, 18, 20], Stoltz and Lugosi [32], and Cahn [4]. The original result of Foster and Vohra shows that if players base their actions on a calibrated forecast of the other players' actions then convergence to correlated equilibria takes place in the above-mentioned sense. Kakade and Foster [26] take these ideas further and

\footnotetext{
${ }^{1}$ Throughout the paper we use the word strategy for both the distribution $\sigma_{t}^{i}$ played at every time instant $t$, as well as for the overall learning procedure or repeated game strategy. In the terminology of Hart [16], our (repeated) strategy belongs to the class of adaptive heuristics and is to be located between evolutionary dynamics and sophisticated learning dynamics in terms of the sphistication of the players. See Hart [16] and also Fudenberg and Levine [13] for more discussion on this.
} 
show that if all players play according to a best response to a certain common "almost deterministic" well-calibrated forecaster (the existence of which they also prove) then the joint empirical frequencies of play converge not only to the set of correlated equilibria but, in fact, to the convex hull of the set of Nash equilibria. Foster and Young [9, 10] introduce two procedures in which, asymptotically, the joint mixed strategy profiles are within distance $\epsilon$ of the set of Nash equilibria in a fraction of at least $1-\epsilon$ of time, though convergence is not achieved.

On the negative side, Hart and Mas-Colell [21, 22] show that it is impossible to achieve convergence to Nash equilibrium for all games if one is restricted to use stationary strategies that have bounded memory. By "bounded memory" we mean that there is a finite integer $T$ such that each player bases his play only on the last $T$ rounds of play. On the other hand, for every $\epsilon>0$ they show a randomized bounded-memory stationary uncoupled procedure for which the joint empirical frequencies of play converge almost surely to an $\epsilon$-Nash equilibrium.

These results reveal that there is a fine line between what is possible in terms of covergence to Nash and what is not. The present paper intends to add to the filling of this thin gap by exhibiting an uncoupled randomized strategy such that the joint mixed strategy profiles converge, almost surely, to a Nash equilibrium for all games. More precisely, in Theorem 2 we prove convergence to Nash equilibrium for a set of "generic" games that include almost all games in the sense of the Lebesgue measure over the set of all finite normal form games. Theorem 3 establishes the existence of an uncoupled randomized strategy that achieves convergence to an $\epsilon$-Nash equilibrium without any restriction on the game. The procedure is based on a careful modification of the regret testing procedure of Foster and Young [10]. The procedure has an unbounded memory (i.e., as time advances, players have to keep track of longer and longer periods of the past) so that this convergence result does not contradict the impossibility result of Hart and Mas-Colell. Note that almost sure convergence of the joint mixed strategy profiles to a single Nash equilibrium is the strongest notion of convergence considered so 
far and implies all other notions cited above.

In Section 7 we strengthen the result by relaxing the assumption of standard monitoring. In this model of "unknown game" we only require that, after taking an action, players observe their own payoffs but not the other players' actions. In fact, they may not know that they are playing against other players. Hart and Mas-Colell [19] show that convergence of the empirical frequencies of play to the set of correlated equilibria may also be achieved in this way. Foster and Young [10] point out that their result can easily be extended to this model, and in fact, it is their idea that we use in Section 7.

The rest of the paper is organized as follows. Section 2 introduces notation and the experimental regret testing procedure. Section 3 contains some basic properties of the procedure. In Section 4 we show that the empirical frequencies converge to the convex hull of the set of $\epsilon$-Nash equilibria (Theorem 1). Section 5 contains the main result: the mixed strategy profiles of an "annealed" and "localized" version of experimental regret testing converges to the set of Nash equilibria (Theorem 2). Section 7 deals with the case in which the players only observe their own payoffs but not the other players' actions. Section 8 contains the proofs of some key lemmas.

\section{Preliminary definitions}

We consider $N$-player normal form games, where with a slight abuse of notation $N$ also denotes the set of players $\{1, \ldots, N\}$. $S_{i}$ denotes player $i$ 's space of pure strategies with cardinality $K_{i}=\# S_{i}$, and $S=\times_{i \in N} S_{i}$ denotes the space of pure strategy profiles with cardinality $K=\sum_{i \in N} K_{i} ; \Sigma_{i}$ denotes the set of probability measures (or mixed strategies) on $S_{i}, \Sigma=\times_{i \in N} \Sigma_{i}$ denotes the space of mixed strategy profiles. Let also $S_{-i}=\times_{j \neq i} S_{j}$ and $\Sigma_{-i}=\times_{j \neq i} \Sigma_{j}$, and for $J \subset N, S_{J}=\times_{i \in J} S_{i}$ and $\Sigma_{J}=\times_{i \in J} \Sigma_{i}$.

Given $N$ and each $K_{i}$ finite, we identify a game with a point in euclidean space $\gamma \in R^{\kappa N}$, where $\kappa=\prod_{i=1}^{N} K_{i}$. We also denote by $\gamma^{i} \in R^{\kappa}$ the payoff array of player $i$ and, by slight abuse of notation, also the payoff function of player $i$ at game $\gamma$. Without loss of generality, we may assume that all payoffs take values in $[0,1]$ so that the space of games reduces to $[0,1]^{\kappa N}$. 
Let $B^{i}(\gamma) \subset \Sigma$ denote the graph of $i$ 's best reply correspondence at $\gamma$ and $B_{\epsilon}^{i}(\gamma) \subset \Sigma$ the graph of $i$ 's $\epsilon$-best reply correspondence; $\mathcal{N}(\gamma)=\cap_{i \in N} B^{i}(\gamma)$ denotes the set of Nash equilibria and $\mathcal{N}_{\epsilon}(\gamma)=\cap_{i \in N} B_{\epsilon}^{i}(\gamma)$ the set of $\epsilon$-Nash equilibria of $\gamma$. Let $\mathcal{N}_{\epsilon}^{c}(\gamma)=\Sigma \backslash \mathcal{N}_{\epsilon}(\gamma)$ denote its complement in $\Sigma$; we will often suppress the argument $\gamma$. Unless otherwise noted, $\mu$ denotes uniform probability measure over either $\Sigma$ or $[0,1]^{\kappa N}$.

The following learning dynamics is based on the regret testing dynamics of Foster and Young [10] and coincides with it when $\lambda=0$.

Definition 1 EXPERIMENTAL REGRET TESTING with parameters $(T, \rho, \lambda)$, where $T \in N, \rho \in R_{+}$, and $\lambda \in(0,1)$, is defined by the following algorithm. 1. Initialization: Set $t=0$. Each player chooses $\sigma_{0}^{i} \in \Sigma_{i}$ uniformly at random.

2. Loop:

(a) Each player plays according to $\sigma_{t}^{i} \in \Sigma_{i}$ for $T \geq 1$ periods, where in each of the $T$ periods a strategy $s_{\tau}^{i} \in S_{i}$ is chosen according to the distribution $\sigma_{t}^{i}$

(b) Each player computes its vector of average regrets over the $T$ periods

$$
r_{t, k}^{i}=\frac{1}{T} \sum_{\tau=t+1}^{t+T}\left(\gamma^{i}\left(k, s_{\tau}^{-i}\right)-\gamma^{i}\left(s_{\tau}\right)\right), \quad k=1, \ldots, K_{i}
$$

where $s_{\tau}=\left(s_{\tau}^{1}, \ldots, s_{\tau}^{N}\right)$ is the $N$-tuple of pure strategies played by the $N$ players at round $\tau$ and $s_{\tau}^{-i}$ is the $N-1$-tuple obtained from $s_{\tau}$ by excluding $s_{\tau}^{i}$.

(c) Each player chooses $\sigma_{t+T}^{i} \in \Sigma_{i}$ as follows: if $r_{t, k}^{i} \geq \rho$ for some $k=1, \ldots, K_{i}$, then randomly select $\sigma_{t+T}^{i} \in \Sigma_{i}$ according to the uniform distribution over $\Sigma_{i}$. If $r_{t, k}^{i}<\rho$ for all $k=1, \ldots, K_{i}$, then with probability $1-\lambda$ set $\sigma_{t+T}^{i}=\sigma_{t}^{i}$ and with probability $\lambda$ randomly select $\sigma_{t+T}^{i} \in \Sigma_{i}$ according to the uniform distribution over $\Sigma_{i}$.

(d) Set $t=t+T$ and repeat the loop.

In words, experimental regret testing with parameters $(T, \rho, \lambda)$ is defined by an updating algorithm, where every $T$ periods each player computes its vector of recent average regrets. If one of the components exceeds $\rho$, then a 
new strategy is drawn from the uniform distribution on the player's strategy simplex, and this strategy is played for the next $T$ periods. If, on the other hand, none of the components exceeds $\rho$, then, with probability $1-\lambda$, it continues to play according to the previous strategy for further $T$ periods, and, with probability $\lambda$, a new strategy is drawn from the uniform distribution on the strategy simplex and is played for the next $T$ periods.

Note that the procedure of experimental regret testing is uncoupled in the sense that the actions of each player only depend on the players' own past payoffs, though a certain amount of coordination is required since we assume that all players use the same parameters $(T, \rho, \lambda)$ and that the intervals of length $T$ over which the players don't change their mixed strategy are synchronized.

The difference of this dynamics from the regret testing dynamics of Foster and Young is that in our case, with a small positive probability $\lambda$, players select a new strategy even if their current strategy does not lead to regrets above the threshold $\rho$. This ensures that there is some amount of experimentation by all the players throughout the learning process.

\section{Properties of experimental regret testing}

We state some key properties of experimental regret testing that will be used throughout the paper. The proofs are all in Section 8.

One of the key properties of experimental regret testing needed to prove such convergence is that the process of joint mixed strategy profiles $\sigma_{0}, \sigma_{T}, \sigma_{2 T}, \ldots$ is a geometrically mixing Markov chain, as summarized in the following lemma. Denote by $\mu$ the uniform probability measure over the set $\Sigma$ of mixed strategy profiles.

Lemma 1 The stochastic process $\left\{\sigma_{t}\right\}, t=0, T, 2 T, \ldots$, defined by experimental regret learning with $0<\lambda<1$, is a recurrent and irreducible $\left(L^{1}\right)$ Markov chain satisfying Doeblin's condition. In particular, for any measurable set $A \subset \Sigma$,

$$
P(\sigma \rightarrow A) \geq \lambda^{N} \mu(A)
$$


for every $\sigma \in \Sigma$ where $P(\sigma \rightarrow A)=P\left\{\sigma_{(m+1) T} \in A \mid \sigma_{m T}=\sigma\right\}$ denotes the transition probabilities of the Markov chain. (Here $m$ is an arbitrary nonnegative integer.)

An immediate corollary is the following (see, e.g., Meyn and Tweedie [29, Theorem 16.2.4]).

Corollary 1 For $m=0,1,2, \ldots$ let $P_{m}$ denote the distribution of $\sigma_{m}$, that is, $P_{m}(A)=P\left\{\sigma_{m T} \in A\right\}$. Then there exists a unique probability distribution $\pi$ over $\Sigma$ (the stationary distribution of the Markov process) such that

$$
\sup _{A}\left|P_{m}(A)-\pi(A)\right| \leq\left(1-\lambda^{N}\right)^{m}
$$

where the supremum is taken over all measurable sets $A \subset \Sigma$.

The main idea behind Foster and Young's heuristics is that, after a not very long search period, by pure chance, the joint mixed strategy profile $\sigma_{m T}$ will be an $\epsilon$-Nash equilibrium, and then, since all players have a small expected regret, the process gets stuck with this value for a much longer time than the search period. The main technical result needed to justify such a statement is summarized in Lemma 3 which will imply that the length of the search period is negligible compared to the length of time the process spends in an $\epsilon$-Nash equilibrium. A similar result was used by Foster and Young [10] for the case of two players.

Throughout the paper we work with generic games in the following sense. Given a game $\gamma \in[0,1]^{\kappa N}$, we say a game $\gamma^{\prime} \in[0,1]^{\kappa^{\prime} N}$ is a pure subgame of $\gamma$ if $S^{\prime} \subset S, \kappa^{\prime}=\prod_{i \in N} K_{i}^{\prime}$, where $K_{i}^{\prime}=\# S_{i}^{\prime} \geq 1$, and where the payoffs are the ones induced by $\gamma$, that is, $\gamma^{\prime}=\gamma_{\mid S^{\prime}}$. For an arbitrary set $J \subset N$ and arbitrary mixed strategy profile $\sigma^{J} \in \Sigma_{J}$, let $\gamma_{\sigma^{J}}$, denote the subgame where players in $J$ play the fixed strategy $\sigma^{J}$. We call an $N$-player normal form game $\gamma \in[0,1]^{\kappa N}$ generic if every pure subgame has only regular Nash equilibria and for every pure subgame $\gamma^{\prime}$ of $\gamma$, we have for almost every mixed strategy profile $\sigma^{J} \in \Sigma_{J}, J \subset N$, that the subgame $\gamma_{\sigma^{J}}^{\prime}$ of $\gamma^{\prime}$ also only has regular Nash equilibria. The notion of regular Nash equilibrium we use is as 
in Ritzberger [30] or van Damme [33]; essentially we require that the system of equations defining a given equilibrium be invertible.

Lemma 2 Almost every game $\gamma \in[0,1]^{\kappa N}$ is generic.

Let $\mathcal{N}_{\epsilon}^{c}(\gamma)=\Sigma \backslash \mathcal{N}_{\epsilon}(\gamma)$ denote the complement of the set of $\epsilon$-Nash equilibria. The next lemma is essential for the convergence results.

Lemma 3 Let $\gamma \in[0,1]^{\kappa N}$ be a generic $N$-player normal form game. Then there exist positive constants $c_{1}, c_{2}$ such that, for all sufficiently small $\rho>0$, if $\rho>\epsilon$, the $N$-step transition probabilities of experimental regret testing satisfy

$$
P^{(N)}\left(\mathcal{N}_{\rho}^{c} \rightarrow \mathcal{N}_{\rho}\right) \geq c_{1} \rho^{c_{2}}
$$

(where we use the notation $P^{(N)}(A \rightarrow B)=P\left\{\sigma_{(m+N) T} \in B \mid \sigma_{m T} \in A\right\}$ for the $N$-step transition probabilities).

One more technical result is needed before we state the main properties of experimental regret testing.

The next basic proposition shows that after sufficiently many rounds of play the distribution of the joint mixed strategies $\sigma$ concentrates in the neighborhood of the set of Nash equilibria. It extends the main result of Foster and Young $[10]$ to generic games of an arbitrary number of players.

Proposition 1 Let $\gamma \in[0,1]^{\kappa N}$ be a generic $N$-player normal form game. There exists a positive number $\epsilon_{0}$ such that for all $\epsilon<\epsilon_{0}$ the following holds: there exist positive constants $c_{1}, \ldots, c_{5}$ such that if the experimental regret testing procedure is used with parameters

$$
\rho \in\left(\epsilon, \epsilon+\epsilon^{c_{1}}\right), \quad \lambda \leq c_{2} \epsilon^{c_{3}}, \quad \text { and } \quad T \geq-\frac{1}{2(\rho-\epsilon)^{2}} \log \left(c_{4} \epsilon^{c_{3}}\right)
$$

then for all $M \geq \log (\epsilon / 2) / \log \left(1-\lambda^{N}\right)$,

$$
P_{M}\left(\mathcal{N}_{\epsilon}^{c}\right)=P\left\{\sigma_{M T} \notin \mathcal{N}_{\epsilon}\right\} \leq \epsilon
$$




\section{Convergence of empirical frequencies}

Next we turn to the first main result of this paper that concerns the long-term joint empirical frequencies of play. Specifically, we show that if all players play according to the experimental regret testing procedure described above, then the joint empirical frequencies of play converge almost surely to a mixed strategy profile $\bar{\sigma}$ that is in the convex hull of $\epsilon$-Nash equilibria. The precise statement is given in Theorem 1.

Recall that, for each $\tau=1,2, \ldots$, we denote by $s_{\tau}^{i} \in S_{i}$ the pure strategy played by the $i$ th player. Recall that $s_{\tau}^{i}$ is drawn randomly according to the mixed strategy $\sigma_{m T}^{i}$ whenever $\tau \in\{m T+1, \ldots,(m+1) T\}$. The $N$ tuple of pure strategies played at time $\tau$ is $s_{\tau}$. Consider the joint empirical distribution of plays $\widehat{P}_{t}$ defined by

$$
\widehat{P}_{t}(s)=\frac{1}{t} \sum_{\tau=1}^{t} I_{s_{\tau}=s} \quad s \in S .
$$

The next theorem shows that if all players play according to experimental regret testing then the joint empirical frequencies of play converge, almost surely, to a fixed mixed strategy profile in the convex hull of the set of $\epsilon$-Nash equilibria. Denote the convex hull of a set $A$ by $\operatorname{co}(A)$.

Theorem 1 Let $\gamma \in[0,1]^{\kappa N}$ be a generic $N$-player normal form game. For every $\epsilon>0$ there exists a choice of the parameters $(T, \rho, \lambda)$ such that there is $a \bar{\sigma} \in \operatorname{co}\left(\mathcal{N}_{\epsilon}\right)$ such that the joint empirical frequencies of play of experimental regret testing satisfy

$$
\lim _{t \rightarrow \infty} \widehat{P}_{t} \rightarrow \bar{\sigma} \quad \text { almost surely. }
$$

Proof. First observe that by martingale convergence, for every $s \in S$,

$$
\widehat{P}_{t}(s)-\frac{1}{t} \sum_{\tau=1}^{t} \sigma_{\tau}(s) \rightarrow 0 \text { almost surely. }
$$

Therefore, it suffices to prove convergence of $\frac{1}{t} \sum_{\tau=1}^{t} \sigma_{\tau}(s)$. Since $\sigma_{\tau}$ is unchanged during periods of length $T$, we obviously have

$$
\lim _{t \rightarrow \infty} \frac{1}{t} \sum_{\tau=1}^{t} \sigma_{\tau}=\lim _{M \rightarrow \infty} \frac{1}{M} \sum_{m=1}^{M} \sigma_{m T} .
$$


By Lemma 1 the process $\left\{\sigma_{m T}\right\}_{m=0}^{\infty}$ is a recurrent and irreducible Markov chain, so the ergodic theorem for Markov chains (see, e.g., [29]) implies that there exists a $\bar{\sigma} \in \Sigma$ such that

$$
\lim _{M \rightarrow \infty} \frac{1}{M} \sum_{m=1}^{M} \sigma_{m T}=\bar{\sigma} \quad \text { almost surely. }
$$

It remains to show that $\bar{\sigma} \in \operatorname{co}\left(\mathcal{N}_{\epsilon}\right)$. By the ergodic theorem, in fact, $\bar{\sigma}=$ $\int_{\Sigma} \sigma d \pi$ where $\pi$ is the (unique) stationary distribution of the Markov process.

Let $\epsilon^{\prime}<\epsilon$ be a positive number such that

$$
\left\{\sigma \in \Sigma: \exists \sigma^{\prime} \in \operatorname{co}\left(\mathcal{N}_{\epsilon^{\prime}}\right) \text { such that }\left\|\sigma-\sigma^{\prime}\right\|_{1}<\epsilon^{\prime}\right\} \subset \operatorname{co}\left(\mathcal{N}_{\epsilon}\right)
$$

Observe that for a generic game such an $\epsilon^{\prime}$ always exists by part (a) of Lemma 6. In fact, one may choose $\epsilon^{\prime}=\epsilon / c_{3}$ for a sufficiently large positive constant $c_{3}$ (whose value depends on the game).

Now choose the parameters $(T, \rho, \lambda)$ such that $\pi\left(\mathcal{N}_{\epsilon^{\prime}}^{c}\right)<\epsilon^{\prime}$. Lemma 1 guarantees the existence of such a choice.

Clearly,

$$
\bar{\sigma}=\int_{\Sigma} \sigma d \pi=\int_{\mathcal{N}_{\epsilon^{\prime}}} \sigma d \pi+\int_{\mathcal{N}_{\epsilon^{\prime}}^{c}} \sigma d \pi
$$

Since $\int_{\mathcal{N}_{\epsilon^{\prime}}} \sigma d \pi \in \operatorname{co}\left(\mathcal{N}_{\epsilon^{\prime}}\right)$, we have that the $L_{1}$ distance of $\bar{\sigma}$ and $\operatorname{co}\left(\mathcal{N}_{\epsilon^{\prime}}\right)$ satisfies

$$
d_{1}\left(\bar{\sigma}, \operatorname{co}\left(\mathcal{N}_{\epsilon^{\prime}}\right)\right) \leq\left\|\int_{\mathcal{N}_{\epsilon^{\prime}}^{c}} \sigma d \pi\right\|_{1} \leq \int_{\mathcal{N}_{\epsilon^{\prime}}^{c}} d \pi=\pi\left(\mathcal{N}_{\epsilon^{\prime}}^{c}\right)<\epsilon^{\prime}
$$

By the choice of $\epsilon^{\prime}$ we indeed have $\bar{\sigma} \in \operatorname{co}\left(\mathcal{N}_{\epsilon}\right)$.

Remark. (INITIALIZATION). In the definition of experimental regret testing we assumed that each player chooses his initial mixed strategy $\sigma_{0}^{i}$ uniformly at random. The reason for the choice of the uniform distribution is merely simplicity, and it is easy to see that Lemma 1 remains true under the weaker assumption that the distribution of $\sigma_{0}$ is absolutely continuous with respect to the uniform measure on $\Sigma$. This observation will be relevant in Section 5 . 
Remark. (UNCOUPLEDNESS). Theorem 1 guarantees, for any fixed $\epsilon$, the existence of the parameters $\rho, \lambda, T$ such that the empirical frequencies of play converge to $\mathcal{N}_{\epsilon}$. However, it is clear from the proof that these parameters depend not only on $\epsilon$ but also on properties of the game. In a genuinely uncoupled way of play, the players should be able to determine these parameters based solely on the value of $\epsilon$. The arguments of Section 5 show that such a procedure may indeed be constructed.

Remark. (RATES OF CONVERGENCE). The bounds established in Lemma 1 also allow us to estimate the length of play $M T$, as a function of $\epsilon$, to achieve that the joint mixed strategy profile is an $\epsilon$-Nash equilibrium with a probability at least $1-\epsilon$. The bounds reveal that experimental regret testing with appropriately chosen parameters achieves this after $O\left((1 / \epsilon)^{C}\right)$ rounds of play where the constant $C$ depends, in a complicated way, on the properties of the game. However, a closer look at the proof reveals that $C$ is at least proportional with $K=\sum_{i=1}^{N} K_{i}$ (the sum of the number of actions of all players) and therefore the speed of convergence is at least exponentially slow as a function of the number of players and the number of actions of each player. This slow rate of convergence is in sharp contrast with the rates of convergence achievable to approximate correlated equilibria. In fact, it follows from results of Cesa-Bianchi and Lugosi [5] that there exists an uncoupled way of play such that, after $O\left(\epsilon^{-2} \log (K / \epsilon)\right)$ rounds of play the joint empirical frequencies of play form, with probability at least $1-\epsilon$, an $\epsilon$-correlated equilibrium.

\section{Convergence of mixed strategy profiles}

The purpose of this section is to derive a regret-based method that guarantees that the joint mixed strategy profiles $\sigma_{t}, t=1,2, \ldots$ converge almost surely to the set $\mathcal{N}$ of Nash equilibria of a generic game. Thus, we not only claim convergence of the empirical frequencies of plays but also of the actual mixed strategy profiles $\sigma_{t}$. Also, we show convergence to $\mathcal{N}$ and not only to the convex hull $\operatorname{co}\left(\mathcal{N}_{\epsilon}\right)$ of all $\epsilon$-Nash equilibria for a fixed $\epsilon$. Actually, our 
proposed method guarantees convergence of $\left\{\sigma_{t}\right\}$ to just one Nash equilibrium, though in case of multiple Nash equilibria the limiting equilibrium may depend on the actual (random) realization of the sequence of plays.

The basic idea is to "anneal" experimental regret testing such that first it is used with some parameters $\left(T_{1}, \rho_{1}, \lambda_{1}\right)$ for a number $M_{1}$ of periods of length $T_{1}$, then change the parameters to $\left(T_{2}, \rho_{2}, \lambda_{2}\right)$ (by increasing $T$ and decreasing $\rho$ and $\lambda$ properly), use experimental regret testing for a number $M_{2} \gg M_{1}$ of periods (of length $T_{2}$ ), etc. However, this is not sufficient to guarantee almost sure convergence as at each change of parameters the process is reinitialized and therefore there is an infinite set of indices $t$ such that $\sigma_{t}$ is far away from any Nash equilibrium. The solution we propose is a careful modification of experimental regret testing that guarantees that for any $\epsilon, \sigma_{t} \notin \mathcal{N}_{\epsilon}$ only occurs a finite number of times, almost surely. This is achieved by "localizing" the search after each change of parameters such that each player limits its choice to a small neighborhood of the mixed strategy played right before the change of parameters (unless a player experiences a large regret in which case the search is extended again to the whole simplex).

Another challenge we must face is that the values of the parameters of the procedure (i.e., $T_{\ell}, \rho_{\ell}, \lambda_{\ell}$, and $M_{\ell}, \ell=1,2, \ldots$ ) cannot depend on the parameters of the game, since by requiring uncoupledness we must assume that the players only know their payoff function but not those of the other players.

Next we define the annealed localized experimental regret testing process. To this end, let $\epsilon_{1}>\epsilon_{2}>\cdots$ be a decreasing sequence of positive numbers such that $\sum_{\ell=1}^{\infty} \epsilon_{\ell}<\infty$.

For each $\ell=1,2, \ldots$ define

$$
\rho_{\ell}=\epsilon_{\ell}+\epsilon_{\ell}^{\ell}, \quad \lambda_{\ell}=\epsilon_{\ell}^{\ell}, \quad \text { and } \quad T_{\ell}=\left\lceil-\frac{1}{2 \epsilon_{\ell}^{2 \ell}} \log \left(\epsilon_{\ell}^{\ell}\right)\right\rceil \text {. }
$$

Introduce also

$$
M_{\ell}=2\left\lceil\frac{\log \frac{2}{\epsilon_{\ell}}}{\log \frac{1}{1-\lambda_{\ell}}}\right\rceil .
$$

and denote by $D_{\infty}(X, \epsilon)$ the $L_{\infty}$-ball of radius $\epsilon$ around $X \subset \Sigma$. 
Definition 2 AnNeAled localized experimental REgret TESTING.

1. Initialization: Each player chooses $\sigma_{0}^{i} \in \Sigma_{i}$ uniformly at random.

2. Loop: There are different regimes indexed by $\ell=1,2, \ldots$ In the $\ell$-th regime, each player plays according to experimental regret testing with parameters $\left(T_{\ell}, \rho_{\ell}, \lambda_{\ell}\right)$ during $M_{\ell}$ periods of length $T_{\ell}$ with the only modification that in step (c) of experimental regret testing, if $\max _{k=1, \ldots, K_{i}} r_{t, k}^{i}<\rho_{\ell-1}$, then the set $\Sigma_{i}$ is replaced by $D_{\infty}\left(\sigma_{[\ell]}^{i}, \sqrt{\epsilon_{\ell}}\right)$ where $\sigma_{[\ell]}^{i}$ is the mixed strategy played by player $i$ at the end of the $\ell-1$-st regime.

Observe that the procedure is fully uncoupled as the only parameter of the procedure is the sequence $\left\{\epsilon_{\ell}\right\}$ which is independent of the properties of the game.

The main result of this section is the following theorem which establishes almost sure convergence of the procedure described above to Nash equilibria.

Theorem 2 Let $\gamma \in[0,1]^{\kappa N}$ be a generic $N$-player normal form game and let $\epsilon_{1}>\epsilon_{2}>\cdots$ be a sequence of positive numbers such that $\sum_{\ell=1}^{\infty} \epsilon_{\ell}<\infty$. If each player plays according to annealed localized experimental regret testing, then the sequence of joint mixed strategy profiles converges almost surely and

$$
\lim _{t \rightarrow \infty} \sigma_{t} \in \mathcal{N} \quad \text { almost surely. }
$$

In case of multiple Nash equilibria the value of the limit may depend on the randomization used in the procedure.

ProOf. The theorem is a quite straightforward consequence of Lemmas 1 , 6 , and the Borel-Cantelli lemma. First note that the parameters $\left(T_{\ell}, \rho_{\ell}, \lambda_{\ell}\right)$ are defined such that for all sufficiently large $\ell$, they satisfy the conditions of Lemma 1 for $\epsilon=\epsilon_{\ell}$. Since $\sum_{\ell} \epsilon_{\ell}<\infty$, by Lemma 1 and the Borel-Cantelli lemma, with probability one, there exist at most a finite number of indices $\ell$ such that at the end of the $\ell$-th regime the joint mixed strategy profile $\sigma_{t}$ is not in $\mathcal{N}_{\epsilon_{\ell}}$. Localization, part (c) of Lemma 6 , and again the Borel-Cantelli lemma guarantees that, with probability one, for all sufficiently large $\ell$, all values of $\sigma_{t}$ in the $\ell$-th regime are within distance $2 \sqrt{\epsilon_{\ell}}$ of a Nash equilibrium. 
Part (c) of Lemma 6 guarantees that for all sufficiently large $\ell$ there is always at least one Nash equilibrium that is not excluded from the search. Since $\epsilon_{\ell} \rightarrow 0$, the process indeed converges to a Nash equilibrium with probability one.

\section{$6 \quad$ Non-generic games}

All results presented up to this point require the game to be generic in the sense specified above. However, since almost all games are generic (with respect to the Lebesque measure over the set $[0,1]^{\kappa N}$ of all games), it is easy to construct a randomized uncoupled procedure such that convergence to an $\epsilon$-Nash equilibrium is achieved for all games.

Theorem 3 Let $\gamma \in[0,1]^{\kappa N}$ be an arbitrary $N$-player normal form game and let $\epsilon>0$. There exists an uncoupled randomized learning procedure such that the joint mixed strategy profiles converge almost surely to a profile $\sigma \in \Sigma$ that is an $\epsilon-N$ ash equilibrium of $\gamma$.

Proof. The idea is that before starting to play, each player slightly perturbes the values of his payoff function and then plays as if his payoff were the perturbed values. For example, define, for each player $i \in N$ and pure strategy profile $s \in S$,

$$
\tilde{\gamma}^{i}(s)=\gamma^{i}(s)+U_{i, s}
$$

where the $U_{i, s}$ are i.i.d. random variables uniformly distributed in the interval $[-\epsilon, \epsilon]$. Clearly, the perturbed game $\tilde{\gamma}$ is generic, with probability one. Therefore, if all players play according to annealed localized experimental regret testing described in Section 5 but based on the payoffs of $\tilde{\gamma}$, then by Theorem 2 the joint mixed strategy profiles $\sigma_{t}$ converge, with probability one, to a Nash equilibrium of $\tilde{\gamma}$. However, since for all $i \in N$ and $s \in S$ we have $\left|\tilde{\gamma}^{i}(s)-\gamma^{i}(s)\right|<\epsilon$, every Nash equilibrium of $\tilde{\gamma}$ is an $\epsilon$-Nash equilibrium of $\gamma$. 
Remark. (NASH CONVERGENCE FOR ALL GAMES). Even though we only prove convergence to $\epsilon$-Nash equilibria in the case of non-generic games, it seems plausible that by a refinement of the same idea as in Theorem 3, it is possible to achieve almost sure convergence to Nash equilibria. The idea is that in annealed localized experimental regret testing, each time the parameters $\left(\rho_{\ell}, \lambda_{\ell}, T_{\ell}\right)$ are updated, the payoffs of the game $\gamma$ are perturbed by a new noise $U_{(i, s), \ell}$ whose magnitude decreases with $\ell$ at an appropriately calibrated way. However, the details of the proof of Nash convergence of such a procedure are quite tedious and we do not work them out here.

\section{Unknown games}

Next we show that all the results shown up to this point extend easily to the significantly more general model where the actions of each player can depend only on past own payoffs, without seeing the actions taken by the rest of the players. This model is sometimes referred to as "unknown game" as the players need not be aware of any characteristics of the game, like, for example, the number of overall players or the number of actions other players can choose from. The setup is closely related to the multi-armed bandit problem where, at each time instance, a player chooses an action and receives a reward but cannot check what reward he would have obtained had he chosen some other action (see, e.g., Auer, Cesa-Bianchi, Freund, and Schapire [1]).

Formally, a strategy for player $i$ is now a sequence of functions that, at time $t$, assign a mixed strategy $\sigma_{t}^{i}$ to the payoff function $\gamma^{i}$, the history of payoffs $\left(\gamma^{i}\left(s_{1}\right), \gamma^{i}\left(s_{2}\right), \ldots, \gamma^{i}\left(s_{t-1}\right)\right)$, and the randomizing variable $\chi_{i, t}$. Just

as before, at time $t$, player $i$ chooses action $s_{t}^{i}$ randomly according to the mixed strategy $\sigma_{t}^{i}$.

Foster and Young [10] already observe that their regret testing procedure can be modified so that the strategy becomes feasible in the unknown game model. Their idea extends in a straightforward way to our modifications as well. In order to adjust the procedures of experimental regret testing and annealed localized experimental regret testing, just note that the only place 
in which the players look at the past is when they calculate the regrets $r_{t, k}^{i}$ in (1). However, each player may estimate his regret in a simple way. The idea is that, at each time instant, player $i$ flips a biased coin and if the outcome is head (whose probability is very small), then instead of choosing an action according to the mixed strategy $\sigma_{t}^{i}$, he chooses one uniformly. At these time instants, the player collects sufficient information to estimate the regret with respect to each fixed action $k \in K_{i}$.

To formalize this idea, consider a period between times $(m-1) T+1$ and $m T$ and denote $t=(m-1) T$. During this period, player $i$ draws $n_{i}$ samples for each $k=1, \ldots, K_{i}$ actions. Formally, define the random variables $U_{i, \tau} \in\left\{0,1, \ldots, K_{i}\right\}$, where, for $\tau$ between $(m-1) T+1$ and $m T$, for each $k=1, \ldots, K_{i}$, there are exactly $n_{i}$ values of $\tau$ such that $U_{i, \tau}=k$, and all such configurations are equally probable; for the remaining $\tau, U_{i, \tau}=0$. (In other words, for each $k=1, \ldots, K_{i}, n_{i}$ values of $\tau$ are chosen randomly, without replacement, such that these values are disjoint for different $k$ 's.) Then, at time $\tau$, player $i$ draws an action $s_{\tau}^{i}$ as follows: conditionally on the past up to time $\tau-1$,

$$
s_{\tau}^{i} \begin{cases}\text { is distributed as } \sigma_{\tau}^{i} & \text { if } U_{i, \tau}=0 \\ \text { equals } k & \text { if } U_{i, \tau}=k\end{cases}
$$

The regret $r_{t, k}^{i}$ may be estimated by

$$
\widehat{r}_{t, k}^{i}=\frac{1}{n_{i}} \sum_{\tau=t+1}^{t+T} I_{U_{i, \tau}=k} \gamma^{i}\left(k, s_{\tau}^{-i}\right)-\frac{1}{T-K_{i} n_{i}} \sum_{\tau=t+1}^{t+T} \gamma^{i}\left(s_{\tau}\right) I_{U_{i, \tau}=0}
$$

$k=1, \ldots, K_{i}$. Observe that $\widehat{r}_{t, k}^{i}$ only depends on the past payoffs experienced by player $i$ and therefore these estimates are feasible in the unknown game model.

In order to show that the estimated regrets work in this case, we only need to establish an analog of inequality (4) for the deviations of the estimated regret. This is done in the next lemma.

Lemma 4 Assume that in a certain period of length $T$, the expected regret $E\left[r_{m T, k}^{i} \mid s_{1}, \ldots, s_{m T}\right]$ of player $i$ is at most $\epsilon$. Then, for a sufficiently small 
$\epsilon$, with the choice of parameters of Proposition 1,

$$
P\left\{\widehat{r}_{m T, k}^{i} \geq \rho\right\} \leq c T^{-1 / 3}+\exp \left(-T^{1 / 3}(\rho-\epsilon)^{2}\right) .
$$

Proof. We show that, with large probability, $\widehat{r}_{m T, k}^{i}$ is close to $r_{m T, k}^{i}$. To this end, note first that

$$
\left|\frac{1}{T-K_{i} n_{i}} \sum_{\tau=t+1}^{t+T} \gamma^{i}\left(s_{\tau}\right) I_{U_{i, \tau}=0}-\frac{1}{T} \sum_{\tau=t+1}^{t+T} \gamma^{i}\left(s_{\tau}\right)\right| \leq 2 \frac{\sum_{i=1}^{N} K_{i} n_{i}}{T} .
$$

On the other hand, observe that, if there is no time instant $\tau$ for which $U_{i, \tau}=1$ and $U_{j, \tau}=1$ for some $j \neq i$, then,

$$
\frac{1}{n_{i}} \sum_{\tau=t+1}^{t+T} I_{U_{i, \tau}=k} \gamma^{i}\left(k, s_{\tau}^{-i}\right)
$$

is an unbiased estimate of

$$
\frac{1}{T} \sum_{\tau=t+1}^{t+T} \gamma^{i}\left(k, s_{\tau}^{-i}\right)
$$

obtained by random sampling. The probability that no two players sample at the same time is at most

$$
T N^{2} \max _{i, j \in N} \frac{K_{i} n_{i}}{T} \frac{K_{j} n_{j}}{T}
$$

and by Hoeffding's inequality [23] for an average of a sample taken without replacement,

$$
\widehat{P}\left\{\left|\frac{1}{n_{i}} \sum_{\tau=t+1}^{t+T} I_{U_{i, \tau}=k} \gamma^{i}\left(k, s_{\tau}^{-i}\right)-\frac{1}{T} \sum_{\tau=t+1}^{t+T} \gamma^{i}\left(k, s_{\tau}^{-i}\right)\right|>\alpha\right\} \leq e^{-2 n_{i} \alpha^{2}}
$$

where $\widehat{P}$ denotes the distribution induced by the random variables $U_{i, \tau}$. Putting everything together,

$P\left\{\hat{r}_{m T, k}^{i} \geq \rho\right\} \leq T N^{2} \max _{i, j \in N} \frac{K_{i} n_{i}}{T} \frac{K_{j} n_{j}}{T}+\exp \left(-2 n_{i}\left(\rho-\epsilon-2 \frac{\sum_{i=1}^{N} K_{i} n_{i}}{T}\right)^{2}\right)$ 
Choosing $n_{i}=O\left(T^{1 / 3}\right)$, the first term on the right-hand side is of order $T^{-1 / 3}$ and $\sum_{i=1}^{N} K_{i} n_{i} / T=O\left(T^{-2 / 3}\right)$ becomes negligible compared to $\rho-\epsilon$ which concludes the statement.

Thus, in the unknown game case, the estimate of inequality (4) can be replaced by that of Lemma 4 . It is easy to see by inspecting the proofs that the rest of the arguments go through without modification, and therefore the results of Theorems 1, 2, and 3 are true in this more general model as well.

Remark. (BAYESIAN GAMES). The unknown game model can be adapted to encompass the case of Bayesian games, i.e., where payoffs depend on strategy profiles chosen as well as players' types. The latter are assumed to be drawn by nature from a finite set and according to a fixed distribution. We only need to require that (i) agents observe their own types and can condition their strategies on those types, and (ii) the game is repeated such that every period nature newly selects the types according to the given distribution. For every block of $T$ periods, agents play fixed conditional strategies, which are resampled if regrets over the previous $T$ periods exceed the regret threshold and are kept unchanged otherwise (up to the experimentation probability $\lambda$ ). Given that the performance of the conditional strategies is (unbiasedly) estimated during play, the present approach does not assume players to have any priors concerning nature's move, but rather to obtain them through repeated play. Players here are quite naive with respect to other players' strategies and types, yet play converges to Bayesian Nash equilibria, in the different senses of Theorems 1, 2, and 3. This is to be contrasted with the belief-based learning approaches, such as, for example, Jordan [24, 25], Dekel, Fudenberg, and Levine [6], or also Kalai and Lehrer [27], Fudenberg and Levine [11], and Nachbar [28].

\section{Proofs}

Proof of Lemma 1. To see that the process is a Markov chain, note that at each $m=0,1,2, \ldots, \sigma_{m T}$ depends only on $\sigma_{(m-1) T}$ and the regrets $r_{(m-1) T, k}^{i}$ $\left(k=1, \ldots, K_{i}, i \in N\right)$. It is clearly $L^{1}$ since $\sigma_{m, k} \in[0,1]$ for all $k, m$, 
it is irreducible since at each $0, T, 2 T, \ldots$, the probability of reaching some $\sigma_{m T}^{\prime} \in A$ for any open set $A \subset \Sigma$ from any $\sigma_{(m-1) T} \in \Sigma$ is strictly positive when $\lambda>0$, and it is recurrent since $E\left[\sum_{m=0}^{\infty} \mathbf{1}_{\left\{\sigma_{m T} \in A\right\}} \mid \sigma_{0} \in A\right]=\infty$ for all $\sigma_{0} \in A$. The Doeblin condition follows simply from the presence of the "exploration parameter" $\lambda$ in the definition of experimental regret testing. In particular, with probability $\lambda^{N}$ every player chooses a mixed strategy randomly and conditioned on this event, the distribution of $\sigma_{m T}$ is uniform.

Proof of Lemma 2. Harsanyi [15] shows that almost every game has a finite (and odd) number of Nash equilibria all of which are regular. Fix the number of players and strategies and let $[0,1]^{\kappa N}$ be the corresponding space of normal form games. Clearly, for any $S^{\prime} \subset S$ we have that, for almost every $\gamma \in[0,1]^{\kappa N}$, the associated pure subgame $\gamma^{\prime}$ of $\gamma$ has finitely many equilibria, all regular. Since $S$ is finite, there are finitely many $S^{\prime} \subset S$ and hence finitely many pure subgames $\gamma^{\prime}$ of $\gamma$. Intersecting over all of these leaves almost all games in $[0,1]^{\kappa N}$ with the property that all pure subgames have finitely many equilibria, all regular.

Next, we show that for almost every game $\gamma \in[0,1]^{\kappa N}$, given $J \subset N$, we have that for almost every profile $\sigma^{J} \in \Sigma_{J}$, the subgame $\gamma_{\sigma^{J}}$ has all equilibria regular. (Notice that if all equilibria are regular then there can only be finitely many of them.) Moreover, since we can view $\gamma$ as the pure subgame of another game, this will prove the general case as well. Fix $J \subset N$ and consider the map $\varphi_{J}:[0,1]^{\kappa N} \rightarrow \Sigma_{J}$ defined by

$$
\varphi_{J}(\gamma)=\left\{\sigma^{J} \in \Sigma_{J}: \gamma_{\sigma^{J}} \text { has nonregular Nash equilibria }\right\}
$$

Since checking whether an equilibrium is nonregular reduces to evaluating the Jacobian of an algebraic function, it is easy to see that this map is semi-algebraic (see Bochnak, Coste, and Roy [3, Prop. 2.2.4]). Therefore, its discontinuities lie on a closed lower-dimensional subset of $[0,1]^{\kappa N}$ such that there are finitely many connected components on which it is continuous (see Schanuel, Simon, and Zame [31] or Blume and Zame [2]). Moreover, if $\varphi_{J}$ is semi-algebraic and takes a set of values $E$ with $\mu(E)>0$ at some point $\bar{\gamma}$ in the interior of a component on which it is continuous, then there must exist 
an open set $E_{0} \subset E$ such that $E_{0} \subset \varphi_{J}(\gamma)$ for any $\gamma$ in an open neighborhood of $\bar{\gamma}$. In other words, for fixed $\sigma_{J} \in E_{0}$, the game $\gamma_{\sigma^{J}}$ has nonregular Nash equilibria for any $\gamma \in G_{0}$, where $G_{0} \subset[0,1]^{\kappa N}$ is an open neighborhood of $\bar{\gamma}$. But since we can view each game $\gamma_{\sigma^{J}}$ as a game in $[0,1]^{\kappa_{J} c N_{J^{c}}}$, and since, in particular, all games in an open neighborhood of $\bar{\gamma} \in[0,1]^{\kappa N} \operatorname{span}$ a corresponding open neighborhood of games in $[0,1]^{\kappa_{J^{c}} N_{J^{c}}}$ around $\bar{\gamma}_{\sigma^{J}}$, (notice that $\sigma_{J} \in E_{0}$ is fixed), we would have that all games in such a neighborhood of $\bar{\gamma}_{\sigma^{J}}$ are degenerate, which is impossible. Hence, it must be the case that if $\varphi_{J}$ takes a set of values with positive measure, it must be at a game where $\varphi_{J}$ is discontinuous. But this can only happen on a lower dimensional set of measure zero and hence, for almost every game $\gamma \in[0,1]^{\kappa N}$, and for any $J \subset N$, we have that for almost every profile $\sigma^{J} \in \Sigma_{J}$, the subgame $\gamma_{\sigma^{J}}$ has all Nash equilibria regular.

LEMmas 5 AND 6 . The proof of Lemma 3 is based on two lemmas. Lemma 5 is concerned with the probabilities of moving from a situation where exactly $J<N$ agents have expected regret less than or equal to $\rho$ (and are playing a profile that is not part of an $\rho$-Nash equilibrium of $\gamma$ ) to a situation where $J-1$ or less agents have expected regret less than or equal to $\rho$. Specifically, it shows that with positive probability, bounded away from zero, the $(N-J)$ agents with expected regret greater than $\rho$ will select a strategy such that (at least) one of the agents in $J$ will also have expected regret greater than $\rho$ in the next period. This is expressed using the sets $C_{\epsilon}^{J}\left(\sigma^{J}\right)$ defined below.

Lemma 6 shows some basic properties of the volume and geometric structure of $\epsilon$-Nash equilibria. Recall that for $J \subset N, \Sigma_{J}=\times_{i \in J} \Sigma_{i}$. Without loss we assume $K_{i} \geq 2, i \in N$.

Lemma 5 Let $\gamma \in[0,1]^{\kappa N}$ be a generic $N$-player normal form game with $K_{i} \geq 2, i \in N$, let $J \subset N$ with $J^{c}=N \backslash J \neq \emptyset$, and let

$$
C_{\epsilon}^{J}\left(\sigma^{J}\right)=\left\{\sigma^{J^{c}} \in \Sigma_{J^{c}}:\left(\sigma^{J}, \sigma^{J^{c}}\right) \in \cap_{i \in J} B_{\epsilon}^{i}\right\}
$$

be the set of profiles in $\Sigma_{J^{c}}$ to which $\sigma^{J} \in \Sigma_{J}$ is a joint $\epsilon$-best reply by the players in $J, \epsilon \geq 0$. Then there exists $\delta(J)>0$ and a positive number $\epsilon_{0}>0$ 
such that for all $\epsilon<\epsilon_{0}$,

$$
\sup _{\sigma^{J}} \mu_{\Sigma_{J^{c}}}\left(C_{\epsilon}^{J}\left(\sigma^{J}\right)\right) \leq 1-\delta(J)<1
$$

where the supremum is taken over all $\sigma^{J} \in \Sigma_{J}$ that are not part of an $\epsilon-N a s h$ equilibrium profile of $\gamma$.

Proof. For an arbitrary set $J \subset N$ and arbitrary mixed strategy profile $\sigma^{J} \in \Sigma_{J}$, let $\gamma_{\sigma^{J}} \in[0,1]^{\kappa_{J}(N-J)}$, where $\kappa_{J}=\Pi_{i \notin J} K_{i}$, denote the subgame where players in $J$ play the fixed strategy $\sigma^{J}$. (Basically this reduces to a game between the players in $J^{c}$.)

First we show the statement for $\epsilon=0$. To simplify notation, we drop the subscript $\epsilon$ whenever $\epsilon=0$. Fix $J \subset N$ with $J^{c} \neq \emptyset$ and consider the correspondence $\eta\left(\sigma^{J^{c}}\right)$ that maps $\sigma^{J^{c}}$ to the set of Nash equilibria of the subgame $\gamma_{\sigma^{3 c}}$. This correspondence is semi-algebraic since it is the composition of two semi-algebraic maps, namely, the map mapping strategy profiles $\sigma^{J^{c}} \in \Sigma_{J^{c}}$ to subgames $\gamma_{\sigma^{J^{c}}} \in[0,1]^{\kappa_{J^{c} J}}$ (this map is convex combinations of pure strategy payoffs) with the Nash correspondence $\mathcal{N}\left(\gamma_{\sigma^{J c}}\right)$ mapping subgames $\gamma_{\sigma^{J^{c}}}$ to Nash equilibria of $\gamma_{\sigma^{J^{c}}}$. Therefore its discontinuities lie on a closed lower-dimensional subset of $\Sigma_{J^{c}}$ such that there are finitely many connected components on which it is continuous (see Schanuel, Simon and Zame [31] or Blume and Zame [2]). Moreover, by our genericity assumption it takes finitely many values for almost every profile $\sigma^{J^{c}} \in \Sigma_{J^{c}}$. This means that there exists a component $D \subset \Sigma_{J^{c}}$ and $\delta_{0}>0$ such that $\eta$ is continuous on $D$, takes finitely many values on a dense subset of $D$, and $\mu_{\Sigma_{J^{c}}}(D)>\delta_{0}$.

To prove the lemma, suppose the claim is false. Suppose there exists a sequence of strategy profiles $\left\{\sigma^{J, n}\right\} \subset \Sigma_{J}$ such that

(i) for every $n, \sigma^{J, n}$ is not part of Nash profile of $\gamma$,

(ii) $\lim _{n \rightarrow \infty} \mu_{\Sigma_{J^{c}}}\left(C^{J}\left(\sigma^{J, n}\right)\right)=1$.

Because $\Sigma_{J}$ is compact, there exists a convergent subsequence $\left\{\sigma^{J, n_{k}}\right\} \subset \Sigma_{J}$ such that (i) and (ii) hold for the corresponding elements. Let $\bar{\sigma}^{J} \in \Sigma_{J}$ be the limit of this subsequence, then $\mu_{\Sigma_{J c}}\left(C^{J}\left(\bar{\sigma}^{J}\right)\right)=1$. This means that for almost every $\sigma^{J^{c}} \in \Sigma_{J^{c}}, \bar{\sigma}^{J} \in \eta\left(\sigma_{J^{c}}\right)$. Because $\eta$ is semi-algbraic and upper 
hemi-continuous, (it is the composition of an upper hemi-continuous correspondence with a continuous map), if it takes the value $\bar{\sigma}^{J}$ almost everywhere on $\Sigma_{J^{c}}$, it must take it everywhere on $\Sigma_{J^{c}}$, i.e., $\bar{\sigma}_{J} \in \eta\left(\sigma_{J^{c}}\right)$ for all $\sigma_{J^{c}} \in \Sigma_{J^{c}}$, in particular $\bar{\sigma}_{J}$ is part of a Nash profile of $\gamma$.

Hence, we may assume without loss that besides (i) and (ii), the sequence $\left\{\sigma^{J, n}\right\}$ also satisfies

(iii) $\lim _{n \rightarrow \infty} \sigma^{J, n}=\bar{\sigma}^{J}$,

(iv) for every $n, \mu_{\Sigma_{J^{c}}}\left(C^{J}\left(\sigma^{J, n}\right)\right)<\mu_{\Sigma_{J c}}\left(C^{J}\left(\sigma^{J, n+1}\right)\right)<1$.

This implies that there exists a sequence of subsets $\left\{E_{n}\right\}=C^{J}\left(\sigma^{J, n}\right) \subset \Sigma_{J^{c}}$ with $\mu_{\Sigma_{J^{c}}}\left(E_{n}\right) \uparrow 1$ such that, for every $n$, the correspondence $\eta$ takes the value $\sigma^{J, n}$ on $E_{n}$, i.e., $\sigma^{J, n} \in \eta\left(\sigma^{J^{c}}\right)$ for all $\sigma^{J^{c}} \in E_{n}$. But then there must exist a set $E$ of positive measure such that $\eta$ takes values arbitrarily close to $\bar{\sigma}^{J}$ on $E$ (by property (iii) above). But this is impossible since on a set of measure one $\eta$ is continuous and takes finitely many values of which $\bar{\sigma}^{J}$ is one of them.

Let now $\epsilon>0$. Suppose that the statement is false, i.e., suppose that for any $\epsilon>0, \sup _{\sigma^{J}} \mu_{\Sigma_{J^{c}}}\left(C_{\epsilon}^{J}\left(\sigma^{J}\right)\right)=1$, where the supremum is taken over all $\sigma^{J} \in \Sigma_{J}$ that are not part of an $\epsilon$-Nash equilibrium profile of $\gamma$. This implies that there is a set $E \subset \Sigma_{J^{c}}$ of strictly positive measure $(\geq \delta(J)$ from the case above with $\epsilon=0)$ such that for any $\sigma^{J^{c}} \in E, \sigma^{J} \in \eta_{\epsilon}\left(\sigma^{J^{c}}\right)$ for any $\epsilon>0$, and at the same time $\sigma^{J} \notin \eta\left(\sigma^{J^{c}}\right)$. Again, this contradicts the fact that $\eta_{\epsilon}$ is semi-algebraic, upper hemi-continuous, and compact-valued.

Lemma 6 Let $\gamma \in[0,1]^{\kappa N}$ be a generic $N$-player normal form game. Then there exist positive constants $c_{1}, \ldots, c_{8}$ such that for all sufficiently small $\epsilon>0$

(a) $D_{\infty}\left(\mathcal{N}, c_{1} \epsilon\right) \subset \mathcal{N}_{\epsilon} \subset D_{\infty}\left(\mathcal{N}, c_{2} \epsilon\right), i \in N$,

(b) $c_{3} \epsilon^{c_{4}} \leq \mu\left(\mathcal{N}_{\epsilon}\right) \leq c_{5} \epsilon, i \in N$.

(c) if $\sigma \in \mathcal{N}_{\epsilon}$, then $D_{\infty}\left(\sigma, c_{6} \epsilon\right) \cap \mathcal{N} \neq \emptyset$.

(d) if $\rho>\epsilon$ and $\rho / \epsilon-1$ is sufficiently small, then $\mu\left(\mathcal{N}_{\rho} \backslash \mathcal{N}_{\epsilon}\right) \leq c_{7}(\rho-\epsilon)^{c_{8}}$.

Proof. (a) Fix $\gamma \in[0,1]^{\kappa N}$ generic and let

$$
\varphi^{i}(\sigma)=\max _{s_{k}^{i} \in S_{i}} \gamma^{i}\left(s_{k}^{i}, \sigma^{-i}\right)-\gamma^{i}(\sigma),
$$


where $\gamma^{i}(\sigma)=\sum_{\nu \in S} \gamma_{\nu}^{i} \prod_{j \in N} \sigma_{\nu_{j}}^{j}$ denotes player $i$ 's payoff function. Notice that $\varphi^{i}$ is semi-algbraic and Lipschitz continuous, where the Lipschitz constant depends only on parameters of the game. Recall $D_{\infty}(\mathcal{N}, \epsilon)=\{\sigma \in \Sigma$ : $\left.\|\sigma-\bar{\sigma}\|_{\infty} \leq \epsilon, \bar{\sigma} \in \mathcal{N}\right\}$ and $\mathcal{N}_{\epsilon}=\left\{\sigma \in \Sigma: \varphi^{i}(\sigma) \leq \epsilon, i \in N\right\}$. By genericity of $\gamma$, the set $\mathcal{N}$ consists of a finite number of regular Nash equilibria, so that the set $\mathcal{N}_{\epsilon}$ can be written as the union of a finite number of neighborhoods, each of which is defined by a finite number of nicely behaved hypersurfaces. More precisely, there exists a positive number $\epsilon_{0}$ such that for any $\epsilon<\epsilon_{0}$, we can write

$$
\mathcal{N}_{\epsilon}=\cup_{\bar{\sigma} \in \mathcal{N}} U(\bar{\sigma} ; \epsilon)
$$

where the sets $U(\bar{\sigma} ; \epsilon), \bar{\sigma} \in \mathcal{N}$, satisfy

(i) $U(\bar{\sigma} ; \epsilon)=\left\{\sigma \in \Sigma: \gamma^{i}\left(s_{k}^{i}, \sigma^{-i}\right)-\gamma^{i}(\sigma) \leq \epsilon\right.$, for all $\left.s_{k}^{i} \in \operatorname{supp}(\bar{\sigma})\right\}$,

(ii) the sets $U(\bar{\sigma} ; \epsilon)$ are pairwise disjoint and, are defined by a finite number of hypersurfaces (of dimension $K-2$; recall $\operatorname{dim} \Sigma=K-1$ ) of bounded curvature that all intersect transversally; moreover, except for the hypersurfaces defining $\Sigma$, which are fixed, all the others are parameterized by $\epsilon$ such that the Hausdorff distance $\mathrm{d}(\Sigma \backslash U(\bar{\sigma}, \epsilon), \bar{\sigma})$ is strictly increasing in $\epsilon$ for $\epsilon$ small.

Because the equations $\gamma^{i}\left(s_{k}^{i}, \sigma^{-i}\right)-\gamma^{i}(\sigma)=\epsilon, s_{k}^{i} \in \operatorname{supp}(\bar{\sigma})$, that bound the sets $U(\bar{\sigma} ; \epsilon)$, vary smoothly with $\epsilon$, it follows that $\mathrm{d}(\Sigma \backslash U(\bar{\sigma}, \epsilon), \bar{\sigma})$ is increasing and Lipschitz continuous in $\epsilon$. Moreover, the genericity assumption implies that the gradient of the functions $h_{s_{k}^{i}}(\sigma)=\gamma^{i}\left(s_{k}^{i}, \sigma^{-i}\right)-\gamma^{i}(\sigma), s_{k}^{i} \in$ $\operatorname{supp}(\bar{\sigma})$, is not the zero vector at $\bar{\sigma}$. Writing the distance (locally) between $\bar{\sigma}$ and the $\sigma$ 's satisfying $h_{s_{k}^{i}}(\sigma)=\epsilon$ as $\left\|\frac{\nabla h_{s_{k}^{2}}}{\left\|\nabla h_{s_{k}^{2}}\right\|}\right\|$, we obtain that the slope of the Hausdorff distance $\mathrm{d}(\Sigma \backslash U(\bar{\sigma}, \epsilon), \bar{\sigma})$ with respect to $\epsilon$ is positive and bounded away from zero. Thus there exist positive constants $C_{1}<C_{2}$ such that $D_{\infty}\left(\bar{\sigma}, C_{1} \epsilon\right) \subset U(\bar{\sigma}, \epsilon) \subset D_{\infty}\left(\bar{\sigma}, C_{2} \epsilon\right)$. Taking $c_{1}, c_{2}$ to be respectively the minimum and maximum over all such constants for the different Nash equilibria yields $D_{\infty}\left(\mathcal{N}, c_{1} \epsilon\right) \subset \mathcal{N}_{\epsilon} \subset D_{\infty}\left(\mathcal{N}, c_{2} \epsilon\right)$.

(b) This follows immediately given the statement and proof of (a). Since $\bar{\sigma}$ is a point in $\Sigma$, we have $\epsilon^{K-1} \leq \mu\left(D_{\infty}(\bar{\sigma}, \epsilon)\right) \leq(2 \epsilon)^{K-1}$ depending on 
whether $\bar{\sigma}$ is in the interior or on the boundary of $\Sigma$. In particular, we have,

$$
\left(c_{1} \epsilon\right)^{K-1} \leq \mu\left(D_{\infty}\left(\mathcal{N}, c_{1} \epsilon\right)\right) \leq \mu\left(\mathcal{N}_{\epsilon}\right) \leq \mu\left(D_{\infty}\left(\mathcal{N}, c_{2} \epsilon\right)\right) \leq\left(2 c_{2} \epsilon\right)^{K-1},
$$

and we can take $c_{3}=c_{1}^{K-1}, c_{4}=K-1$, and $c_{5}=\left(2 c_{2}\right)^{K-1}$.

(c) From (a) we have for any $\epsilon>0$ small, $D_{\infty}\left(\mathcal{N}, c_{1} \epsilon\right) \subset \mathcal{N}_{\epsilon} \subset D_{\infty}\left(\mathcal{N}, c_{2} \epsilon\right)$. Hence, if $\sigma \in \mathcal{N}_{\epsilon}$ then $\sigma \in D_{\infty}\left(\mathcal{N}, c_{2} \epsilon\right)$. Taking $c_{6}=2 c_{2}$ we have $D_{\infty}\left(\sigma, c_{6} \epsilon\right) \cap$ $\mathcal{N} \neq \emptyset$.

(d) From (a) we have for any $\rho, \epsilon>0$ small, $D_{\infty}\left(\mathcal{N}, c_{1} \epsilon\right) \subset \mathcal{N}_{\epsilon}$ and $\mathcal{N}_{\rho} \subset D_{\infty}\left(\mathcal{N}, c_{2} \rho\right)$, and hence, for $\rho>\epsilon$,

$$
\mathcal{N}_{\rho} \backslash \mathcal{N}_{\epsilon} \subset D_{\infty}\left(\mathcal{N}, c_{2} \rho\right) \backslash D_{\infty}\left(\mathcal{N}, c_{1} \epsilon\right)
$$

where $c_{2} \geq c_{1}$. For the volume we have,

$$
\begin{aligned}
\mu\left(\mathcal{N}_{\rho} \backslash \mathcal{N}_{\epsilon}\right) & \leq \mu\left(D_{\infty}\left(\mathcal{N}, c_{2} \rho\right) \backslash D_{\infty}\left(\mathcal{N}, c_{1} \epsilon\right)\right) \\
& =\mu\left(D_{\infty}\left(\mathcal{N}, c_{2} \rho\right)\right)-\mu\left(D_{\infty}\left(\mathcal{N}, c_{1} \epsilon\right)\right) \\
& =\left(c_{2}(2 \rho)^{K-1}-c_{1}(2 \epsilon)^{K-1}\right)(\# \mathcal{N}) \\
& \leq c_{1} 2^{K-1}(\# \mathcal{N})\left(\rho^{K-1}-\epsilon^{K-1}\right) \\
& \leq c_{5}(\rho-\epsilon)
\end{aligned}
$$

where $c_{5}=c_{1} 2^{K-1}(\# \mathcal{N})<\infty$. The last inequality follows for $\rho / \epsilon-1$ small.

Proof of Lemma 3. Lemma 5 implies that, if there are exactly $J<N$ players who have regret less than $\rho$ and are playing a profile $\sigma^{J} \in \Sigma_{J}$ that is not part of a $\rho$-Nash equilibrium profile, then there is a positive probability, bounded away from zero (uniformly for all possible subsets $J \subset N$; take $\left.\min _{J \subset N} \frac{\delta(J)}{2}\right)$, that the strategy profiles randomly chosen by the players in $J^{c}$ will be such that all players in $J^{c}$ and at least one player in $J$ will have expected regret greater than $\rho$ at the new strategy profile. For the remaining $J-1$ players, there are two possibilities: (a) their joint strategy profile is part of a $\rho$-Nash equilibrium, (b) their joint strategy profile is not part of a $\rho$-Nash equilibrium. Since we are looking for a lower bound for $P^{(N)}\left(\mathcal{N}_{\rho}^{c} \rightarrow \mathcal{N}_{\rho}\right)$, it suffices to follow up on case (b). In case (b), Lemma 5 always applies, and repeatedly following up on those cases, one reaches a situation (after at most 
$N-1$ steps), where all $N$ players randomly sample a new strategy. Applying Lemma 6 at this last step and combining this with the previous, we have that there exists $\delta>0$ such that for every $\rho>0, P^{(N)}\left(\mathcal{N}_{\rho}^{c} \rightarrow \mathcal{N}_{\rho}\right) \geq$ $\delta^{N-1} C_{1} \rho^{C_{2}}$, for some positive constants $C_{1}, C_{2}$. In particular, there exist positive constants $c_{1}, c_{2}$ such that, for any $\rho>0, P^{(N)}\left(\mathcal{N}_{\rho}^{c} \rightarrow \mathcal{N}_{\rho}\right) \geq c_{1} \rho^{c_{2}}$.

Proof of Proposition 1. First note that by Corollary 1,

$$
P_{M}\left(\mathcal{N}_{\epsilon}^{c}\right) \leq \pi\left(\mathcal{N}_{\epsilon}^{c}\right)+\left(1-\lambda^{N}\right)^{M}
$$

so that it suffices to bound the measure of $\mathcal{N}_{\epsilon}^{c}$ under the stationary probability $\pi$. Clearly,

$$
\pi\left(\mathcal{N}_{\rho}\right)=\pi\left(\mathcal{N}_{\rho}^{c}\right) P^{(N)}\left(\mathcal{N}_{\rho}^{c} \rightarrow \mathcal{N}_{\rho}\right)+\pi\left(\mathcal{N}_{\rho}\right) P^{(N)}\left(\mathcal{N}_{\rho} \rightarrow \mathcal{N}_{\rho}\right)
$$

Writing $\pi\left(\mathcal{N}_{\rho}^{c}\right)=1-\pi\left(\mathcal{N}_{\rho}\right)$ and solving for $\pi\left(\mathcal{N}_{\rho}\right)$, we have

$$
\pi\left(\mathcal{N}_{\rho}\right)=\frac{P^{(N)}\left(\mathcal{N}_{\rho}^{c} \rightarrow \mathcal{N}_{\rho}\right)}{1-P^{(N)}\left(\mathcal{N}_{\rho} \rightarrow \mathcal{N}_{\rho}\right)+P^{(N)}\left(\mathcal{N}_{\rho}^{c} \rightarrow \mathcal{N}_{\rho}\right)}
$$

where

$$
\begin{aligned}
P^{(N)}\left(\mathcal{N}_{\rho} \rightarrow \mathcal{N}_{\rho}\right)= & \frac{\pi\left(\mathcal{N}_{\epsilon}\right) P^{(N)}\left(\mathcal{N}_{\epsilon} \rightarrow \mathcal{N}_{\rho}\right)}{\pi\left(\mathcal{N}_{\rho}\right)} \\
& +\frac{\pi\left(\mathcal{N}_{\rho} \backslash \mathcal{N}_{\epsilon}\right) P^{(N)}\left(\mathcal{N}_{\rho} \backslash \mathcal{N}_{\epsilon} \rightarrow \mathcal{N}_{\rho}\right)}{\pi\left(\mathcal{N}_{\rho}\right)} \\
\geq & \frac{\pi\left(\mathcal{N}_{\epsilon}\right) P^{(N)}\left(\mathcal{N}_{\epsilon} \rightarrow \mathcal{N}_{\rho}\right)}{\pi\left(\mathcal{N}_{\rho}\right)}
\end{aligned}
$$

To bound $P^{(N)}\left(\mathcal{N}_{\epsilon} \rightarrow \mathcal{N}_{\rho}\right)$ note that if $\sigma_{m T} \in \mathcal{N}_{\epsilon}$ then the expected regret of all players is at most $\rho$. Since the regret estimates $r_{m T, k}^{i}$ are sums of $T$ independent random variables taking values between 0 and 1 with mean at most $\epsilon$, Hoeffding's inequality [23] implies that

$$
P\left\{r_{m T, k}^{i} \geq \rho\right\} \leq e^{-2 T(\rho-\epsilon)^{2}}, \quad k=1, \ldots, K_{i}, \quad i=1, \ldots, N
$$

Then the probability that there is at least one player $i$ and a strategy $k \leq K_{i}$ such that $r_{m T, k}^{i} \geq \rho$ is bounded by $\sum_{i=1}^{N} K_{i} e^{-2 T(\rho-\epsilon)^{2}}=K e^{-2 T(\rho-\epsilon)^{2}}$. Thus, 
with probability at least $(1-\lambda)^{N}\left(1-K e^{-2 T(\rho-\epsilon)^{2}}\right)$, all players keep playing the same mixed strategy and therefore

$$
P\left(\mathcal{N}_{\epsilon} \rightarrow \mathcal{N}_{\epsilon}\right) \geq(1-\lambda)^{N}\left(1-K e^{-2 T(\rho-\epsilon)^{2}}\right) .
$$

Consequently, since $\rho>\epsilon$, we have $P\left(\mathcal{N}_{\epsilon} \rightarrow \mathcal{N}_{\rho}\right) \geq P\left(\mathcal{N}_{\epsilon} \rightarrow \mathcal{N}_{\epsilon}\right)$ and hence $P^{(N)}\left(\mathcal{N}_{\epsilon} \rightarrow \mathcal{N}_{\rho}\right) \geq(1-\lambda)^{2 N}\left(1-K e^{-2 T(\rho-\epsilon)^{2}}\right)^{N} \geq 1-2 N \lambda-N K e^{-2 T(\rho-\epsilon)^{2}}$

(where we assumed $\lambda \leq 1$ and $K e^{-2 T(\rho-\epsilon)^{2}} \leq 1$ ). Thus, using (3) and the obtained estimate, we have

$$
P^{(N)}\left(\mathcal{N}_{\rho} \rightarrow \mathcal{N}_{\rho}\right) \geq\left(1-2 N \lambda-N K e^{-2 T(\rho-\epsilon)^{2}}\right) \frac{\pi\left(\mathcal{N}_{\epsilon}\right)}{\pi\left(\mathcal{N}_{\rho}\right)}
$$

Next we need to show that, for proper choice of the parameters, $P^{(N)}\left(\mathcal{N}_{\rho}^{c} \rightarrow\right.$ $\mathcal{N}_{\rho}$ ) is sufficiently large. For generic games of $N$ players, this follows from Lemma 3 which asserts that

$$
P^{(N)}\left(\mathcal{N}_{\rho}^{c} \rightarrow \mathcal{N}_{\rho}\right) \geq C_{1} \rho^{C_{2}}
$$

for some positive constants $C_{1}$ and $C_{2}$ that depend on the game. Hence, from (2) we obtain

$$
\pi\left(\mathcal{N}_{\rho}\right) \geq \frac{C_{1} \rho^{C_{2}}}{1-\left(1-2 N \lambda-N K e^{-2 T(\rho-\epsilon)^{2}}\right) \frac{\pi\left(\mathcal{N}_{\epsilon}\right)}{\pi\left(\mathcal{N}_{\rho}\right)}+C_{1} \rho^{C_{2}}}
$$

It remains to estimate the measure $\pi\left(\mathcal{N}_{\epsilon}\right) / \pi\left(\mathcal{N}_{\rho}\right)$. To this end, observe that if $\rho$ is sufficiently small then the ratio $\pi\left(\mathcal{N}_{\rho} \backslash \mathcal{N}_{\epsilon}\right) / \pi\left(\mathcal{N}_{\epsilon}\right)$ is bounded by the ratio of the corresponding Lebesgue measures $\mu\left(\mathcal{N}_{\rho} \backslash \mathcal{N}_{\epsilon}\right) / \mu\left(\mathcal{N}_{\epsilon}\right)$. (Just note that the "density" of $\pi$ decreases by moving away from a Nash equilibrium. More precisely, $\pi$ may not be absolutely continuous with respect to the Lebesgue measure, but one can show that if $\sigma_{1} \in \mathcal{N}_{\rho} \backslash \mathcal{N}_{\epsilon}$ and $\sigma_{2} \in \mathcal{N}_{\epsilon}$ then for a sufficiently small $0<\xi \ll \epsilon$ the $l_{\infty}$ ball of radius $\xi$ centered at $\sigma_{1}$ has a $\pi$-measure less than or equal to that of the same ball centered at $\sigma_{2}$.) The ratio of the volumes of $\mathcal{N}_{\rho} \backslash \mathcal{N}_{\epsilon}$ and $\mathcal{N}_{\epsilon}$ may therefore be bounded by invoking parts $(c)$ and $(d)$ of Lemma 6 . We obtain

$$
\frac{\pi\left(\mathcal{N}_{\rho} \backslash \mathcal{N}_{\epsilon}\right)}{\pi\left(\mathcal{N}_{\epsilon}\right)} \leq \frac{C_{3}(\rho-\epsilon)^{C_{4}}}{C_{5} \epsilon^{C_{6}}}
$$


so that

$$
\frac{\pi\left(\mathcal{N}_{\epsilon}\right)}{\pi\left(\mathcal{N}_{\rho}\right)}=1-\frac{\pi\left(\mathcal{N}_{\rho} \backslash \mathcal{N}_{\epsilon}\right)}{\pi\left(\mathcal{N}_{\rho}\right)} \geq 1-\frac{C_{3}(\rho-\epsilon)^{C_{4}}}{C_{5} \rho^{C_{6}}}
$$

In summary,

$$
\begin{aligned}
& \pi\left(\mathcal{N}_{\epsilon}\right) \\
& \geq \pi\left(\mathcal{N}_{\rho}\right)\left(1-\frac{C_{3}(\rho-\epsilon)^{C_{4}}}{C_{5} \rho^{C_{6}}}\right) \\
& \geq\left(1-\frac{C_{3}(\rho-\epsilon)^{C_{4}}}{C_{5} \rho^{C_{6}}}\right) \frac{C_{1} \rho^{C_{2}}}{1-\left(1-2 N \lambda-N K e^{-2 T(\rho-\epsilon)^{2}}\right)\left(1-\frac{C_{3}(\rho-\epsilon)^{C_{4}}}{C_{5} \rho^{C_{6}}}\right)+C_{1} \rho^{C_{2}}}
\end{aligned}
$$

for some positive constants $C_{1}, \ldots, C_{6}$. Substituting the choices of the parameters $\rho, \lambda, T$ with sufficiently large constants $c_{1}, \ldots, c_{6}$ we have

$$
\pi\left(\mathcal{N}_{\epsilon}^{c}\right) \leq \epsilon / 2 .
$$

If $M$ is so large that $\left(1-\lambda^{N}\right)^{M} \leq \epsilon / 2$, we have $P_{M}\left(\mathcal{N}_{\epsilon}^{c}\right) \leq \epsilon$ as desired.

Acknowledgments. We thank Sergiu Hart, Sham Kakade, Andreu MasColell, and Peyton Young for sharing their view with us on the subject, and also Bill Zame for helpful comments. The first author acknowledges financial support from the Spanish Ministry of Science and Technology, grant SEJ2004-03619, and in form of a Ramon y Cajal fellowship. The second author acknowledges support by the PASCAL Network of Excellence under EC grant no. 506778. The work of the second author was supported by the Spanish Ministry of Science and Technology and FEDER, grant BMF200303324.

\section{References}

[1] P. Auer, N. Cesa-Bianchi, Y. Freund, and R.E. Schapire. The nonstochastic multiarmed bandit problem. SIAM Journal on Computing, 32:48-77, 2002.

[2] L.E. Blume and W.R. Zame. The algebraic geometry of perfect and sequential equilibrium. Econometrica, 62:783-794, 1994. 
[3] J. Bochnak, M. Coste, and M.F. Roy. Real Algebraic Geometry. Springer-Verlag, Berlin, 1998.

[4] A. Cahn. General procedures leading to correlated equilibria. International Journal of Game Theory, 2004.

[5] N. Cesa-Bianchi and G. Lugosi. Potential-based algorithms in on-line prediction and game theory. Machine Learning, 51:239-261, 2003.

[6] E. Dekel, D. Fudenberg, and D. Levine. Learning to play Bayesian games. Games and Economic Behavior, 46:282-303, 2004.

[7] D. Foster and R. Vohra. Calibrated learning and correlated equilibrium. Games and Economic Behaviour, 21:40-55, 1997.

[8] D. Foster and R. Vohra. Regret in the on-line decision problem. Games and Economic Behavior, 29:7-36, 1999.

[9] D.P. Foster and P.H. Young. Learning, hypothesis testing, and Nash equilibrium. Games and Economic Behavior, 45:73-96, 2003.

[10] D.P. Foster and P.H. Young. Regret testing: A simple payoff-based procedure for learning Nash equilibrium. Mimeo, University of Pennsylvania and Johns Hopkins University, 2003.

[11] D. Fudenberg and D. Levine. Steady state learning and Nash equilibrium. Econometrica, 61:547-574, 1993.

[12] D. Fudenberg and D. Levine. Universal consistency and cautious fictitious play. Journal of Economic Dynamics and Control, 19:1065-1089, 1995.

[13] D. Fudenberg and D. Levine. The theory of learning in games. MIT Press, Cambridge MA, 1998.

[14] D. Fudenberg and D. Levine. Universal conditional consistency. Games and Economic Behavior, 29:104-130, 1999. 
[15] J. C. Harsanyi. Oddness of the number of equilibrium points: a new proof. International Journal of Game Theory, pages 235-250, 1973.

[16] S. Hart. Adaptive Heuristics. Technical report, The Hebrew University of Jerusalem, 2004.

[17] S. Hart and A. Mas-Colell. A simple adaptive procedure leading to correlated equilibrium. Econometrica, 68:1127-1150, 2000.

[18] S. Hart and A. Mas-Colell. A general class of adaptive strategies. Journal of Economic Theory, 98:26-54, 2001.

[19] S. Hart and A. Mas-Colell. A reinforcement procedure leading to correlated equilibrium. In G. Debreu, W. Neuefeind, and W. Trockel, editors, Economic Essays: A Festschrift for Werner Hildenbrand, pages 181-200. Srpinger, New York, 2002.

[20] S. Hart and A. Mas-Colell. Regret-based continuous-time dynamics. Games and Economic Behavior, 45:375-394, 2003.

[21] S. Hart and A. Mas-Colell. Uncoupled dynamics do not lead to Nash equilibrium. American Economic Review, 93:1830-1836, 2003.

[22] S. Hart and A. Mas-Colell. Stochastic uncoupled dynamics and Nash equilibrium. Technical report, The Hebrew University of Jerusalem, 2004.

[23] W. Hoeffding. Probability inequalities for sums of bounded random variables. Journal of the American Statistical Association, 58:13-30, 1963.

[24] J.S. Jordan. Bayesian learning in normal form games. Games and Economic Behavior, 3:60-81, 1991.

[25] J.S. Jordan. Bayesian learning in repeated games. Games and Economic Behavior, 9:8-20, 1995. 
[26] S.M. Kakade and D.P. Foster. Deterministic calibration and Nash equilibrium. In Proceedings of the 17th Annual Conference on Learning Theory. Springer, 2004.

[27] E. Kalai and E. Lehrer Rational learning leads to Nash equilibrium. Econometrica, 61:1019-1045, 1993.

[28] J.H. Nachbar Prediction, optimization, and learning in repeated games. Econometrica, 65:275-309, 1997.

[29] S.P. Meyn and R.L. Tweedie. Markov chains and stochastic stability. Springer-Verlag, London, 1993.

[30] K. Ritzberger. The theory of normal form games from the differentiable viewpoint. International Journal of Game Theory, 23:207-236, 1994.

[31] Schanuel S.H., L.K. Simon, and W.R. Zame. The algebraic geometry of games and the tracing procedure. In R. Selten, editor, Game Equilibrium Models, II: Methods, Morals, and Markets. Springer Verlag, Berlin, 1991.

[32] G. Stoltz and G.Lugosi. Learning correlated equilibria in games with compact sets of strategies. Technical report, Université Paris-Sud, Orsay, 2004.

[33] E. van Damme. Stability and perfection of Nash equilibria. SpringerVerlag, New York, 1991. 